\title{
Criotolerancia de Semen Equino Congelado con Aditivos en el Diluyente
}

\author{
Cryotolerance of Stallion Semen Frozen with Additives in the Extender \\ Juan Esteban Duque C. ${ }^{1}$, Benjamín A. Rojano², Giovanni Restrepo B. ${ }^{3,4}$
}

\section{Resumen}

Los diluyentes se han constituido como una alternativa para compensar las deficiencias antioxidantes del semen equino durante su congelación. Muchas investigaciones han estado dirigidas a evaluar el efecto de diversos aditivos con propiedades antioxidantes sobre la criotolerancia de los espermatozoides equinos; sin embargo, en limitadas ocasiones se ha relacionado dicha propiedad con la capacidad antioxidante total (CAT) del diluyente. Esta investigación tuvo como objetivo evaluar el efecto de dos aditivos sobre la CAT del diluyente de congelación y la criotolerancia del semen equino. El semen de cinco caballos criollos colombianos se congeló con un diluyente en tres tratamientos: T0: control, T1: quercetina $100 \mu \mathrm{M}, \mathrm{T} 2$ : fosfatos y carbonatos $\left(\mathrm{Na}_{2} \mathrm{HPO}_{4} 0.32 \mathrm{mg} / \mathrm{ml}+\right.$ $\left.\mathrm{KH}_{2} \mathrm{PO}_{4} 0.67 \mathrm{mg} / \mathrm{ml}+\mathrm{K}_{2} \mathrm{CO}_{3} 0.39 \mathrm{mg} / \mathrm{ml}\right)$. Se evaluó la CAT del diluyente mediante la prueba ABTS. En el semen descongelado se evaluó la movilidad y la cinética, la morfología anormal (MA) y la integridad estructural (IEM) y funcional (HOS) de la membrana plasmática de los espermatozoides. Los resultados se analizaron mediante modelos mixtos. Se hallaron valores de CAT ( $\mu$ mol trolox/l) para T0, T1 y T2 de $10890 \pm 210,14260 \pm$ 410 y $14670 \pm 410$, respectivamente. El T2 presentó valores superiores para la mayoría de parámetros de movilidad e integridad de membrana $(\mathrm{p}<0.05)$. Se concluye que la adición de fosfatos y carbonatos incrementa la CAT del diluyente de congelación y mejora la criotolerancia del semen equino.

Palabras clave: diluyente, criopreservación, carbonatos, fosfatos, quercetina

\section{Abstract}

Extenders have been established as an alternative to compensate the antioxidant deficiencies of stallion semen during freezing. Several research works have aimed to evaluate the effect of various additives with antioxidant properties on the equine sperm

\footnotetext{
${ }^{1}$ Facultad de Ciencias Agrarias, Politécnico Colombiano Jaime Isaza Cadavid, Medellín, Colombia

${ }^{2}$ Facultad de Ciencias, Universidad Nacional de Colombia, Medellín, Colombia

${ }^{3}$ Facultad de Ciencias Agrarias, Universidad Nacional de Colombia, Medellín, Colombia

${ }^{4}$ E-mail: grestre0@unal.edu.co
}

Recibido: 14 de julio de 2016

Aceptado para publicación: 11 de noviembre de 2016 
cryotolerance; however, in limited occasions this has been linked with the extender total antioxidant capacity (TAC). This research aimed to evaluate the effect of two additives on freezing extender TAC and stallion semen cryotolerance. Semen of five Colombian Creole horses was frozen in an extender under three treatments: T0: control, T1: quercetin $100 \mu \mathrm{M}, \mathrm{T} 2$ : phosphates and carbonates $\left(\mathrm{Na}_{2} \mathrm{HPO}_{4} 0.32 \mathrm{mg} / \mathrm{ml}+\mathrm{KH}_{2} \mathrm{PO}_{4} 0.67 \mathrm{mg} / \mathrm{ml}+\right.$ $\mathrm{K}_{2} \mathrm{CO}_{3} 0.39 \mathrm{mg} / \mathrm{ml}$ ). TAC of extender was evaluated by the ABTS test. Post-thaw motility and kinetics, abnormal morphology (AM), and structural (MSI) and functional (HOS) plasma membrane integrity were assessed. The results were analysed using mixed models. TAC values ( $\mu$ mol trolox/l) for T0, T1 and T2 of $10890 \pm 210,14260 \pm 410$ and $14670 \pm 410$ were found. The T2 had higher values for most parameters of motility and membrane integrity $(p<0.05)$. It is concluded that the addition of phosphates and carbonates increases the freezing extender TAC and improves stallion semen cryotolerance.

Key words: extender, cryopreservation, carbonates, phosphates, quercetin

\section{INTRODUCCIÓN}

La optimización de los diluyentes para la congelación de semen equino busca garantizar la máxima viabilidad y fertilidad posible del semen criopreservado. Este concepto es fundamental, debido a que la inseminación artificial con semen criopreservado cumple un importante papel en el uso de caballos de fenotipo superior en los programas de mejoramiento genético (Ribeiro et al., 2015). No obstante, la menor fertilidad del semen equino congelado-descongelado se debe a una serie de factores, pudiéndose citar a la toxicidad del plasma seminal, la lesión criogénica a través del estrés osmótico, la criocapacitación, las transiciones de fase de membrana, la peroxidación lipídica y la crioprotección inferior a la óptima (Aspland et al., 2014).

Otros autores han atribuido la reducida criotolerancia del semen equino a factores asociados con el estrés oxidativo, como la pérdida de la actividad de defensa antioxidante (Macias-Garcia et al., 2011), la alta variabilidad en las propiedades antioxidantes entre los reproductores (Aitken et al., 2014) y la generación diferencial de especies reactivas de oxígeno (ERO) entre los espermatozoides no viables o de pobre calidad y aquellos con metabolismo rápido y altos niveles de fosforilación oxidativa (Gibb y Aitken, 2016).
Los diluyentes se han constituido como alternativa para compensar los problemas que ocurren durante la congelación del semen equino, pues se les puede adicionar diversas moléculas con propiedades antioxidantes (Coutinho da Silva et al., 2008; Bustamante et al., 2009; Gibb et al., 2013; Lançoni et al., 2015). La extracción del plasma seminal es un procedimiento rutinario antes de la congelación del semen equino (Guasti et al., 2012), que ocasiona no solo la pérdida de antioxidantes enzimáticos y no enzimáticos (Pesch et al., 2006; Waheed et al., 2013), sino también de macro y microelementos relacionados con la calidad seminal (Pesch et al., 2006). Entre estos, iones como el sodio $(\mathrm{Na})$, potasio $(\mathrm{K})$ y fósforo $(\mathrm{P})$ son esenciales en el balance osmótico del semen (Asadpour, 2012; Abdul-Wahab et al., 2014). Se conoce que el ion fosfato es crucial para la actividad de la adenilato-ciclasa como principal reguladora de la movilidad espermática (Banjoko et al., 2013). Adicionalmente, el potasio juega un papel importante en la activación de las células espermáticas (Asadpour, 2012).

Como alternativa, la adición al diluyente de fuentes de sodio, fósforo, potasio, cloro e incluso magnesio, se ha relacionado con el mejoramiento de la movilidad del semen equino criopreservado (Padilla y Foote, 1991). 
Aditivos como fosfato de sodio $\left(\mathrm{Na}_{2} \mathrm{HPO}_{4}\right)$, fosfato de potasio $\left(\mathrm{KH}_{2} \mathrm{PO}_{4}\right)$ y bicarbonato de sodio $\left(\mathrm{NaHCO}_{3}\right)$ son propios de la composición de diferentes diluyentes para semen equino (Coutinho da Silva et al., 2012); sin embargo, pocas investigaciones se han orientado en evaluar sus efectos particulares. Algunos de estos suplementos podrían tener efecto sobre la actividad antioxidante del diluyente, como es el caso de los fosfatos, cuyas propiedades antioxidantes se cree están basadas en su acción como quelantes (Weilmeier y Regenstein, 2004). La quercetina es uno de los antioxidantes con efectos favorables sobre el semen equino congelado, pues mejora la movilidad y la habilidad de unión a la zona pelúcida, evita la capacitación prematura y reduce la peroxidación lipídica y la fragmentación del ADN de los espermatozoides (McNiven y Richardson, 2006; Gibb et al., 2013).

A pesar de lo anterior, pocas veces se ha considerado el aporte de los antioxidantes y otros suplementos a la capacidad antioxidante total (CAT) del diluyente; no obstante, su importancia ha sido ampliamente demostrada en la criotolerancia del semen de especies como carneros (Yildiz et al., 2015), porcinos (Barranco et al., 2015) y bovinos (Gürler et al., 2015). En equinos, estudios previos han relacionado la CAT del plasma seminal con diferentes parámetros de calidad espermática (Wnuk et al., 2010; Restrepo et al., 2015). La presente investigación tuvo como objetivo evaluar el efecto de varios aditivos (quercetina, fosfatos y carbonatos) sobre la CAT del diluyente de congelación y la criotolerancia del semen equino.

\section{Materiales y Métodos}

Cinco caballos criollos colombianos (Equus caballus), ubicados en el Valle del Aburra (Antioquia, Colombia), se utilizaron para la colecta de 15 eyaculados (tres por animal), mediante el método de la vagina artificial. Los animales estuvieron entre los cua- tro y ocho años de edad, todos en un régimen de una colecta semanal como mínimo, con fertilidad comprobada por crías nacidas vivas y con una condición corporal de 8 (escala 1-9). El semen colectado se diluyó en proporción 1:1 en diluyente EquiPlus ${ }^{\circledR}$ (Minitube, Alemania) a $37^{\circ} \mathrm{C}$ y transportado en un dispositivo de refrigeración Equitainer ${ }^{\circledR}$ (Hamilton Research Inc., EEUU). Se evaluó la concentración espermática mediante un espectrofotómetro Spermacue ${ }^{\circledR}$ (Minitube, Alemania) y la movilidad por microscopía de contraste de fase (Eclipse E200, Nikon Inc., Japón). Solo se procesaron los eyaculados con mínimo de $100 \times 10^{6}$ espermatozoides $/ \mathrm{ml}$ y $60 \%$ de movilidad total.

Para la congelación seminal se utilizó un diluyente a base de caseinatos de sodio, azúcares, antioxidantes, antibióticos, $5 \%$ de $\mathrm{N}, \mathrm{N}$-dimetilformamida (Sigma-Aldrich, EEUU) y $5 \%$ de yema de huevo centrifugada. Como tratamientos se consideró la adición de suplementos: T0: Control; T1: quercetina $100 \mu \mathrm{M}$; T2: fosfatos y carbonatos $\left(\mathrm{Na}_{2} \mathrm{HPO}_{4}\right.$ $0.32 \mathrm{mg} / \mathrm{ml}+\mathrm{KH}_{2} \mathrm{PO}_{4} 0.67 \mathrm{mg} / \mathrm{ml}+\mathrm{K}_{2} \mathrm{CO}_{3}$ $0.39 \mathrm{mg} / \mathrm{ml}$ ). La yema centrifugada se preparó de acuerdo a lo descrito por Nouri et al. (2013), para lo cual se diluyó en una proporción de 3:1 en agua ultrapura y se centrifugó a $1600 \mathrm{~g}$ durante 99 minutos.

El semen se dividió en tres alícuotas, y se centrifugaron a $850 \mathrm{~g}$ por $12 \mathrm{~min}$. El precipitado de cada alícuota se resuspendió aleatoriamente en uno de los tratamientos y se mantuvieron en refrigeración a $5{ }^{\circ} \mathrm{C}$ por una h. Luego se empacó en pajillas para 0.5 $\mathrm{ml}$ en una máquina MRS1 Dual V2 (IMV Technologies, Francia). Las pajillas fueron sometidas a vapores de nitrógeno líquido por 15 min y se almacenaron en un tanque para nitrógeno líquido.

Se evaluó por triplicado la capacidad antioxidante total (CAT) del diluyente correspondiente a cada tratamiento, mediante la prueba ABTS (Arts et al., 2004). Se emplearon $10 \mu$ de semen y $990 \mu 1$ de la solución del radical $\mathrm{ABTS}^{\bullet+}$ (Sigma-Aldrich, EEUU). Lue- 
go de 30 min de reacción a temperatura ambiente y en la oscuridad, se midió en un espectrofotómetro $6405 \mathrm{UV} / \mathrm{Vis}$ (Jenway, EEUU) el cambio en la absorbancia respecto a una solución referencia, compuesta por $10 \mu 1$ de solución buffer y $990 \mu 1$ de la solución del radical $\mathrm{ABTS}^{\bullet+}$. El radical se generó por oxidación de $3.5 \mathrm{mM}$ de ABTS con 1.25 $\mathrm{mM}$ de persulfato de potasio. Después de 24 $\mathrm{h}$ de reacción, se ajustó la absorbancia con PBS a pH 7.4 hasta 0.70 unidades, a una $l$ de $732 \mathrm{~nm} y$ se comparó contra una curva patrón con Trolox ${ }^{\circledR}$ (Sigma-Aldrich, EEUU).

La movilidad y la cinética espermática se evaluaron mediante el sistema $\mathrm{SCA}^{\circledR}$ (Microptic S.L., España) de acuerdo a lo descrito por Restrepo et al. (2013). Se utilizó un microscopio de contraste de fase Eclipse E200 (Nikon, Inc., Japón) con una cámara digital Scout SCA780 (Basler, EEUU). Se evaluó la movilidad total (MT), la movilidad progresiva (MP), la velocidad rectilínea (VSL), curvilínea (VCL) y media (VAP), los índices de linealidad (LIN), rectitud (STR) y oscilación (IO), el desplazamiento lateral de la cabeza (ALH), la frecuencia de batido (BCF), la hiperactividad (HIP) y los movimientos circulares (CIRC). La morfología anormal (MA) se evaluó mediante la tinción con eosina-nigrosina (Barth y Oko, 1989), evaluándose la morfología de 200 espermatozoides en un microscopio de contraste de fase Eclipse E200 (Nikon Inc., Japón).

La integridad funcional de la membrana plasmática (HOS) se evaluó mediante la prueba hipoosmótica descrita por Neild et al. (1999). Para esto, se tomó $100 \mu 1$ de semen y se adicionó a un tubo con $500 \mu \mathrm{l}$ de una solución hipoosmótica de sacarosa 5.4\% (100 $\mathrm{mOsmol} / \mathrm{l})$, donde la mezcla se incubó a $38.5^{\circ} \mathrm{C}$ por $30 \mathrm{~min}$, evaluándose la reacción de 200 espermatozoides por microscopía de contraste de fase Eclipse E200 (Nikon Inc., Japón). La integridad estructural de la membrana plasmática (IEM) se evaluó utilizando el procedimiento descrito por Gamboa et al. (2010), con el kit Live/Dead (Molecular
Probes Inc., EEUU). Para esto, se suspendieron $200 \mu 1$ de semen en solución Hanks Heppes $(\mathrm{HH})$ con $1 \%$ de albúmina sérica bovina (BSA, Sigma Aldrich, EEUU), para una concentración de 20 x $10^{6}$ espermatozoides $/ \mathrm{ml}$. Luego la mezcla se incubó a $37^{\circ} \mathrm{C}$ por $8 \mathrm{~min}$ con $6 \mathrm{mM}$ de SYBR14. Seguidamente se incubó de la misma manera con $0.48 \mathrm{mM}$ de yoduro de propidio (IP). A partir de una muestra de $5 \mu 1$, se realizó el conteo de 200 espermatozoides, mediante un filtro UV-2A de un microscopio E200 con fluorescencia HBO (Nikon Inc., Japón).

Se utilizó un diseño completamente al azar. Los datos se analizaron mediante el ajuste de modelos mixtos. Se incluyó como efecto fijo el tratamiento y como efecto aleatorio el eyaculado anidado dentro del equino. Para asegurar la normalidad de los datos se utilizó la prueba de Shapiro-Wilk y se realizaron transformaciones logarítmicas. Las comparaciones de medias entre tratamientos se realizaron mediante la prueba de Tukey. Todas las evaluaciones se realizaron con el programa SAS 9.2. (SAS Institute Inc., EEUU).

\section{Resultados y Discusión}

Los 15 eyaculados de semen fresco presentaron los siguientes resultados promedio de calidad seminal: volumen (libre de gel) $43.1 \pm 20.7 \mathrm{ml}$, concentración: $229.4 \pm 92.4 \mathrm{x}$ $10^{6}$ espermatozoides $/ \mathrm{ml}$, MT $89.9 \pm 7.9 \%$, MP $56.3 \pm 16.5 \%$, MA $29.2 \pm 5.8$, IE, $83.9 \pm 5.5 \%$ y HOS $67.4 \pm 8.3 \%$. Estos resultados fueron similares a reportes previos de calidad seminal de caballos criollos colombianos (Restrepo et al., 2014; 2016).

Existen resultados divergentes en cuanto a la suplementación del semen con antioxidantes, lo cual se atribuye a factores como la especie, el diluyente utilizado y el tipo y la concentración de los antioxidantes (Sikka, 2004). Por lo tanto, la evaluación de la CAT puede dar mayor información, 
Cuadro 1. Movilidad y cinética espermática (media \pm desviación estándar) de semen de caballos criollos colombianos posdescongelación según el aditivo utilizado en el diluyente $^{1}$

\begin{tabular}{|c|c|c|c|}
\hline Variable $^{2}$ & T0 & $\mathrm{T} 1$ & $\mathrm{~T} 2$ \\
\hline MT & $41.4 \pm 13.6^{\mathrm{a}}$ & $42.0 \pm 12.5^{\mathrm{a}}$ & $58.2 \pm 18.3^{\mathrm{b}}$ \\
\hline MP & $24.4 \pm 11.2^{\mathrm{a}}$ & $23.6 \pm 9.2^{\mathrm{a}}$ & $36.4 \pm 17.1^{\mathrm{b}}$ \\
\hline VCL & $74.8 \pm 11.7^{\mathrm{ab}}$ & $73.9 \pm 12.2^{\mathrm{a}}$ & $77.1 \pm 12.5^{\mathrm{b}}$ \\
\hline VSL & $36.2 \pm 11.1^{\mathrm{a}}$ & $35.5 \pm 10.9^{\mathrm{a}}$ & $40.9 \pm 11.1^{\mathrm{b}}$ \\
\hline VAP & $47.8 \pm 11.4^{\mathrm{a}}$ & $46.4 \pm 11.2^{\mathrm{a}}$ & $54.8 \pm 13.5^{\mathrm{b}}$ \\
\hline LIN & $48.0 \pm 10.4^{\mathrm{a}}$ & $47.6 \pm 10.0^{\mathrm{a}}$ & $52.7 \pm 10.0^{\mathrm{b}}$ \\
\hline STR & $74.8 \pm 8.6^{\mathrm{a}}$ & $75.6 \pm 8.8^{\mathrm{a}}$ & $74.2 \pm 7.3^{\mathrm{a}}$ \\
\hline IO & $63.6 \pm 8.1^{\mathrm{a}}$ & $62.4 \pm 7.5^{\mathrm{a}}$ & $70.7 \pm 8.6^{\mathrm{b}}$ \\
\hline ALH & $3.0 \pm 0.3^{\mathrm{b}}$ & $3.1 \pm 7.5^{\mathrm{b}}$ & $2.9 \pm 0.3^{\mathrm{a}}$ \\
\hline $\mathrm{BCF}$ & $11.3 \pm 1.4^{\mathrm{b}}$ & $12.0 \pm 1.4^{\mathrm{c}}$ & $9.4 \pm 1.3^{\mathrm{a}}$ \\
\hline HIP & $11.5 \pm 6.3^{\mathrm{a}}$ & $12.5 \pm 5.9^{\mathrm{ab}}$ & $13.2 \pm 5.2^{\mathrm{b}}$ \\
\hline CIRC & $20.8 \pm 9.3^{\mathrm{a}}$ & $21.8 \pm 8.2^{\mathrm{a}}$ & $27.0 \pm 9.6^{\mathrm{b}}$ \\
\hline \multicolumn{4}{|c|}{$\begin{array}{l}{ }^{1} \mathrm{~T} 1 \text { : control; } \mathrm{T} 2 \text {; quercetina } 100 \mu \mathrm{M} \text {; T3: fosfatos y carbonatos }\left(\mathrm{Na}_{2} \mathrm{HPO}_{4} 0.32 \mathrm{mg} / \mathrm{ml}+\mathrm{KH}_{2} \mathrm{PO}_{4} 0.67\right. \\
\left.\mathrm{mg} / \mathrm{ml}+\mathrm{K}_{2} \mathrm{CO}_{3} 0.39 \mathrm{mg} / \mathrm{ml}\right) \\
\text { a,b } \\
\left.{ }^{2} \text { Superíndices diferentes dentro de filas denotan diferencia estadística significativa ( } \mathrm{p}<0.05\right) \text {. } \\
\text { VAP: velocidadad media. LIN: índice de linealidad. STR: índice de rectitud. IO: índice de oscilación. } \\
\text { ALH: amplitud lateral de la cabeza. BCF: frecuencia de batida. HIP: hiperactivos. CIRC: movimiento } \\
\quad \text { circular }\end{array}$} \\
\hline
\end{tabular}

biológicamente relevante, que la obtenida a partir de la medición de concentraciones de antioxidantes individuales (Ghiselli et al., 2000). Los valores de la CAT mediante la prueba de ABTS para los diluyentes utilizados fueron de: T0: $10890 \pm 210 \mu \mathrm{mol}$ trolox $/ 1$, $\mathrm{T} 1: 14260 \pm 410 \mu \mathrm{mol}$ trolox/l y T2: $14670 \pm$ $410 \mu \mathrm{mol}$ trolox/l; lo cual muestra un incremento en la CAT del diluyente por la adición de quercetina (T1) y fosfatos y carbonatos (T2). Yildiz et al. (2015), encontraron este mismo efecto mediante la adición de ergotioneina en semen de carneros.

Un total de 225 pajillas de semen congelado fueron descongeladas para la evaluación de los parámetros de calidad seminal (Cuadro 1). En relación a la movilidad y la cinética espermática, se encontró que T2 fue superior para la mayoría de parámetros evaluados $(\mathrm{p}<0.05)$. De otro lado, los resultados para $\mathrm{T} 1$ fueron similares al tratamiento control (T0), siendo solo diferentes para BCF $(\mathrm{p}<0.05)$.

La suplementación con fosfatos y carbonatos (T2), además de producir el mayor incremento de la CAT, generó un evidente mejoramiento de la tolerancia del semen a la congelación. Los fosfatos son utilizados comúnmente por sus propiedades para regular el pH (Perrin y Dempsey, 1974); sin embargo, entre sus propiedades antioxidantes se sabe que podrían actuar como quelantes de ERO (Weilmeier y Regenstein, 2004) y que tienen un muy fuerte efecto antioxidante contra la oxidación de los lípidos, por su unión a iones metálicos que actúan como 


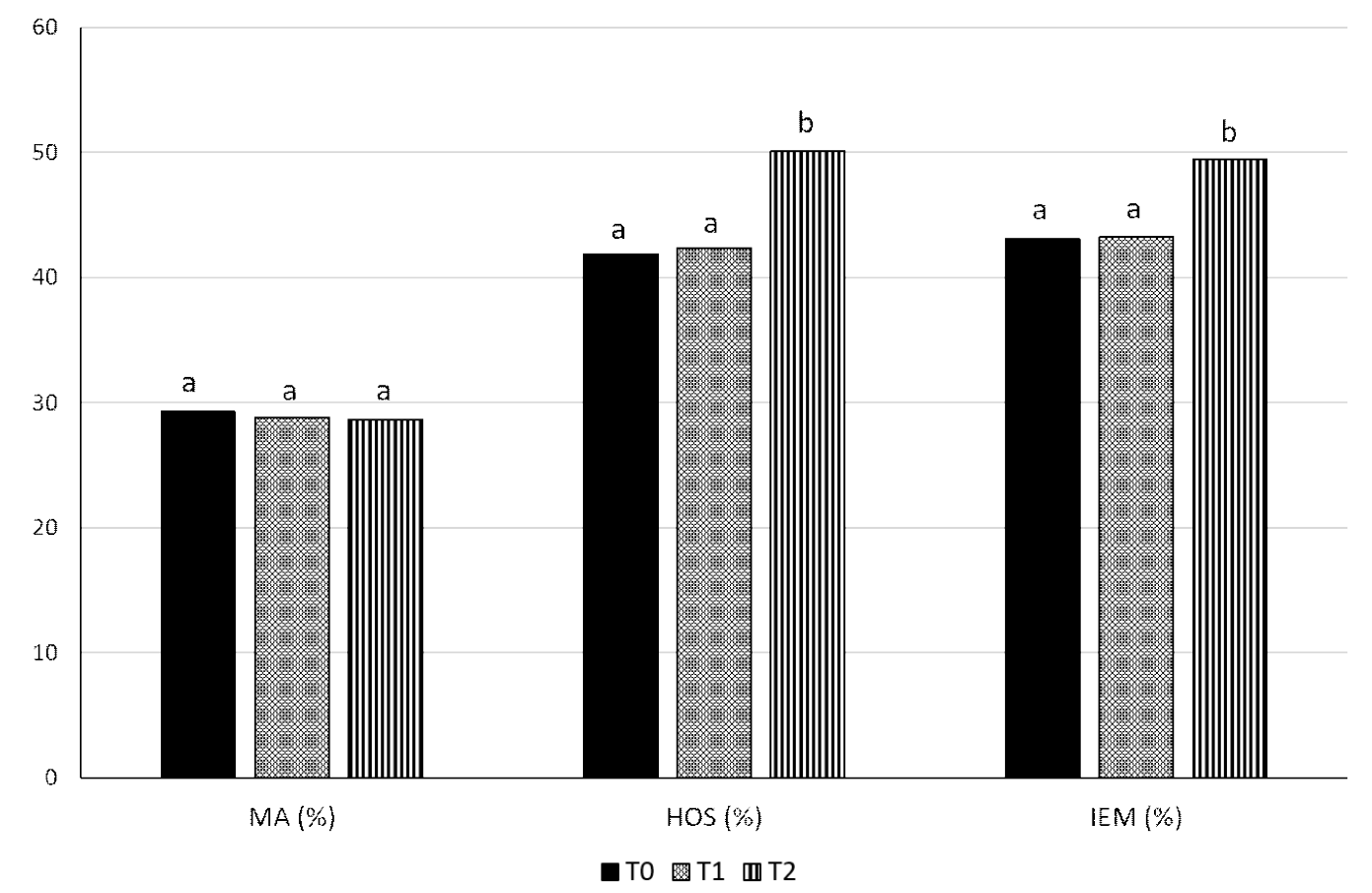

Figura 1. Morfología e integridad de membrana posdescongelación de semen de caballos crio1los colombianos posdescongelación según el aditivo utilizado en el diluyente (T1: control; T2; quercetina $100 \mu \mathrm{M}$; T3: fosfatos y carbonatos $\left[\mathrm{Na}_{2} \mathrm{HPO}_{4} 0.32 \mathrm{mg} / \mathrm{ml}+\right.$ $\left.\left.\mathrm{KH}_{2} \mathrm{PO}_{4} 0.67 \mathrm{mg} / \mathrm{ml}+\mathrm{K}_{2} \mathrm{CO}_{3} 0.39 \mathrm{mg} / \mathrm{ml}\right]\right)$. MA: morfología anormal. HOS: integridad funcional de membrana. IEM: integridad estructural de membrana

catalizadores de la oxidación, lo que les permite la reducción de la peroxidación lipídica (Craig et al., 1996; Kýlýç et al., 2015). Esto último podría explicar la reducción de la alteración estructural y funcional de la membrana plasmática de los espermatozoides, dada la presencia de fosfato de sodio y el fosfato de potasio en el T2 (Figura 1). No se conocen otros reportes del efecto de la adición de fosfatos y carbonatos sobre la CAT del diluyente o el semen. Tabassomi y AlaviShoushtari (2013) encontraron que la suplementación con $0.032 \mathrm{mg} / \mathrm{l}$ de $\mathrm{CuSO}_{4}$ al diluyente para semen de búfalo (Bubalus bubalis) produjo un incremento posdescongelación en la CAT, así como un descenso en el daño del ADN y un aumento en la MP y la vitalidad de los espermatozoides.

Los resultados de calidad seminal encontrados coinciden con los reportados por Padilla y Foote (1991) y Karow et al. (1992), quienes observaron que la adición de potasio al diluyente de congelación mejoró la movilidad espermática en la especie equina y humana, respectivamente. De otro lado, se conoce la importancia del ion fosfato para la actividad de la adenilato-ciclasa como principal regulador de la movilidad espermática (Banjoko et al., 2013), Así mismo, se ha reportado que el potasio juega un papel importante en la activación de las células espermáticas, mientras el sodio y el potasio participan en el establecimiento del equilibrio osmótico del semen (Asadpour, 2012). Adicionalmente, el bicarbonato de sodio también participa en el sistema de regulación de la adenilato-ciclasa como modulador la movilidad (Okamura et al., 1986). Respecto al uso de carbonatos de potasio, se conoce que su adición al medio de congelación permite conservar la movilidad progresiva y la congelabilidad del semen de salmón (Mounib, 1978), lo cual coincidió con lo observado en esta investigación para el T2 (Cuadro 1). 
Abdul-Wahab et al. (2014) encontraron una correlación positiva de los niveles de sodio y potasio con la movilidad de los espermatozoides, sugiriendo que la progresividad del movimiento espermático podría mejorarse con un aumento en la concentración de dichos iones. En esta investigación, el aporte de sodio y potasio a partir de los fosfatos y carbonatos, podría relacionarse con el incremento de la MP para el T2, la cual fue superior al control (T0) en un $12 \%$ $(\mathrm{p}<0.05)$. Por su parte, Kanwal et al. $(2000)$ reportaron una correlación positiva entre los niveles de potasio en el plasma seminal y la movilidad de los espermatozoides de bóvidos. Sin embargo, para la especie ovina, los niveles de potasio se han correlacionado negativamente con la progresividad de los espermatozoides (Abdel-Rahman et al., 2000).

Las diferencias observadas en la mayoría de los parámetros cinéticos evaluados en el semen descongelado fueron originadas por la adición de fosfatos y carbonatos (Cuadro 1). Aunque para el T2 se observó un incremento en la HIP $(p<0.05)$, los valores superiores encontrados para VSL y LIN $(p<0.05)$ son contrarios a los patrones de hiperactivación de espermatozoides equinos reportados por McPartlin et al. (2009), quienes asociaron el movimiento hiperactivo con un descenso en VSL, STR y LIN. Otros cambios considerados como descriptivos de la hiperactividad de los espermatozoides equinos fueron la reducción de MP y BCF, así como el incremento en ALH (Ortgies et al., 2012), los cuales, a excepción de la disminución en BCF $(p<0.05)$, fueron contrarios a lo observado para T2 (Cuadro 1).

En esta investigación no se observó ningún efecto por parte de la quercetina sobre la movilidad y la cinética espermática (Cuadro 1), a excepción de un leve incremento en la BCF $(\mathrm{p}<0.05)$. De igual forma, no hubo diferencias por la adición de quercetina sobre la morfología e integridad de membrana (Figura 1). No obstante, la quercetina (T1) produjo un incremento en la CAT del diluyente $(14260 \pm 410 \mu \mathrm{mol}$ trolox/l) respecto a T0 $(10890 \pm 210 \mu \mathrm{mol}$ trolox/l). Este efecto podría ser explicado por la capacidad de la quercetina para suprimir la formación del ion superóxido, quelatos de hierro e inhibir la formación de radicales peroxi-lipídicos (Afanas'ev et al., 1989). Gibb et al. (2013) hallaron un incremento cercano al $5 \%$ en la movilidad espermática posdescongelación mediante el uso de una concentración mayor de quercetina $(150 \mu \mathrm{M})$; sin embargo, el resultado de MT $(25.3 \pm 2.5 \%)$ fue muy inferior al reportado en el presente trabajo (Cuadro 1), por lo cual es posible considerar un efecto compensatorio del diluyente de congelación, por la presencia de otras moléculas con actividad antioxidante (Bustamante et al., 2009). Otros trabajos con quercetina han encontrado efectos como la reducción del potencial de membrana mitocondrial, la movilidad y la vitalidad de los espermatozoides (Khanduja et al. 2001; Silva et al. 2012).

\section{Conclusiones}

- La adición de fosfatos y carbonatos incrementa la capacidad antioxidante total del diluyente de congelación y mejora la criotolerancia del semen equino.

- La adición de quercetina incrementa la capacidad antioxidante total del diluyente de congelación; sin embargo, bajo las condiciones de este estudio, no tuvo efecto sobre la calidad posdescongelación del semen equino.

\section{Literatura Citada}

1. Abdel-Rahman H, El-Belely M, AlQarawi A, El-Mougy SA. 2000. The relationship between semen quality and mineral composition of semen in various ram breeds. Small Rum Res 38: 45-49. doi: 10.1016/S0921-4488(00)00137-1 
2. Abdul-Wahab R, Daghistani H, Shquirat W, Abdel-Dayem $M$, Mohammad A. 2014. Sodium, potassium, calcium and copper levels in seminal plasma are associated with sperm quality in fertile and infertile men. Biochem Pharmacol 3(4). doi: 10.4172/ 2167-0501.1000141

3. Afanas'ev I, Dcrozhko A, Brodskii A, Kostyuk V, Potapovitch A. 1989. Chelating and free radical scavenging mechanisms of inhibitory action of rutin and quercetin in lipid peroxidation. Biochem Pharmacol 38: 1763-1769. doi: 10.1016/0006-2952(89)90410-3

4. Aitken R, Lambourne S, Gibb Z. 2014. The John Hughes Memorial Lecture: aspects of sperm physiology-oxidative stress and the functionality of stallion spermatozoa. J Equine Vet Sci 34: 1727. doi: 10.1016/j.jevs.2013.10.120

5. Arts M, Dallinga S, Voss H, Haenen $G$, Bast A. 2004. A new approach to assess the total antioxidant capacity using the TEAC assay. Food Chem 88: 567570. doi: 10.1016/j.foodchem.2004.02.008

6. Asadpour R. 2012. Relationship between mineral composition of seminal plasma and semen quality in various ram breeds. Acta Sci Vet 40: 1027.

7. Aspland A, Satake N, Cawdell-Smith A, Collins C. 2014. Assessing novel, plant-based cryopreservation extenders for freezing stallion semen. J Equine Vet Sci 34: 54-55. doi: 10.1016/j.jevs.2013.10.032

8. Banjoko O, Adeselou F. 2013. Seminal plasma $\mathrm{pH}$, inorganic phosphate, total and ionized calcium concentrations in the assessment of human spermatozoa function. J Clin Diagn Res 7: 2483-2486. doi: 10.7860/JCDR/2013/6194.3585

9. Barranco I, Tvarijonaviciute A, PerezPatiño C, Parrilla I, Ceron J, Rodríguez-Martínez H, et al. 2015. High total antioxidant capacity of the porcine seminal plasma (SP-TAC) relates to sperm survival and fertility. Sci Rep 5: 18538. doi: 10.1038/srep18538
10. Barth AD, Oko RJ. 1989. Abnormal morphology of bovine spermatozoa. USA: Iowa University Press. 285 p.

11. Bustamante I, Pederzolli C, Sgaravatti A, Gregory R, Dutra C, Jobim M, Costa $R$. 2009. Skim milk-egg yolk based semen extender compensates for non-enzymatic antioxidant activity loss during equine semen cryopreservation. Anim Reprod 6: 392-399.

12. Coutinho da Silva M, Ferreira $H$, Johnson A. 2008. Effects of tempol and L-ergothioneine on motility parameters of cryopreserved stallion sperm. Anim Reprod Sci. 107: 317-318. doi: 10.1016/ j.anireprosci.2008.05.094

13. Coutinho da Silva M, Seidel G Jr, Squires E, Graham J, Carnevale E. 2012. Effects of components of semen extenders on the binding of stallion spermatozoa to bovine or equine zonae pellucidae. Reproduction 143: 577-585. doi: 10.1530/REP-11-0099.

14. Craig J, Bowers J, Wang X, Selb P. 1996. Inhibition of lipid oxidation in meats by inorganic phosphate and ascorbate salt. J Food Sci 61: 1062-1067. doi: 10.1111/j.1365-2621.1996.tb10933.x

15. Gamboa S, Rodrigues A, Henriques L, Batista C, Ramalho-Santos J. 2010. Seasonal functional relevance of sperm characteristic in equine spermatozoa. Theriogenology 73: 950-958. doi: 10.1016/j.theriogenology.2009.11.023

16. Ghiselli A, Serafini M, Natella F, Scaccini C. 2000. Total antioxidant capacity as a tool to assess redox status: critical view and experimental data. Free Radic Biol Med 29: 1106-1114. doi: 10.1016/S0891-5849(00)00394-4

17. Gibb Z, Aitken R. 2016. The impact of sperm metabolism during in vitro storage: the stallion as a model. Biomed Res Int 2016: 9380609. doi: http://dx.doi.org/ 10.1155/2016/9380609

18. Gibb Z, Butler T, Morris L, Maxwell W, Grupen C. 2013. Quercetin improves the post-thaw characteristics of cryopreserved sex-sorted and 
nonsorted stallion sperm. Theriogenology 79: 1001-1009. doi: 10.1016/j.theriogenology.2012.06.032

19. Guasti P, Monteiro G, Papa F. 2012. Componentes do plasma seminal e sua influência sobre a criopreservação e fertilidade de espermatozoides equinos. Vet Zootec 19: 169-180.

20. Gürler H, Calisici O, Bollwein H. 2015. Inter- and intra-individual variability of total antioxidant capacity of bovine seminal plasma and relationships with sperm quality before and after cryopreservation. Anim Reprod Sci 155: 99-105. doi: 10.1016/j.anireprosci.2015.02.006

21. Kanwal M, Rehman N, Ahmad N, Samad H, Zia-Ur-Rehman N, Akhtar $N$, et al. 2000. Bulk cations and trace elements in the Nili-Ravi buffalo and crossbred cow bull semen. Int J Agric Biol 2: 302-305.

22. Kýlýç B, Simsek A, Claus J, Atýlgan E, Aktas N. 2015. Effects of different end-point cooking temperatures on the efficiency of encapsulated phosphates on lipid oxidation inhibition in ground meat. J Food Sci 80: C2161-C2169. doi: 10.1111/1750-3841.13009

23. Karow A, Gilbert W, Black J. 1992. Effects of temperature, potassium concentration, and sugar on human spermatozoa motility: a cell preservation model from reproductive medicine. Cryobiology 29: 250-254. doi: 10.1016/ 0011-2240(92)90023-U

24. Khanduja K, Verma A, Bhardwaj A. 2001. Impairment of human sperm motility and viability by quercetin is independent of lipid peroxidation. Andrologia 33: 277-281. doi: 10.1046/ j.1439-0272.2001.00432.x

25. Lançoni R, Celeghini E, BianchiAlves M, Santos G, Florez-Rodriguez S, Leite T, Arruda RP. 2015. Use of melatonin and ferulic acid as promoters of cryopreserved equine sperm. Anim Reprod 12: 559 (Abstract).
26. Macias-Garcia B, Gonzalez-Fernandez L, Ortega-Ferrusola C, MorilloRodriguez A, Gallardo-Bolaños $J$, Rodríguez-Martínez H, et al. 2011. Fatty acids and plasmalogens of the phospholipids of the sperm membranes and their relation with the post-thaw quality of stallion spermatozoa. Theriogenology 75: 811-818. doi: 10.1016/j.theriogenology.-2010.10.021

27. McNiven M, Richardson G. 2006. Effect of quercetin on capacitation status and lipid peroxidation of stallion spermatozoa. Cell Preserv Technol 4: 169-177. doi: 10.1089/cpt.2006.4.169.

28. McPartlin L, Suarez S, Czaya C, Hinrichs K, Bedford-Guaus S. 2009. Hyperactivation of stallion sperm is required for successful in vitro fertilization of equine oocytes. Biol Reprod 81: 199-206. doi: 10.1095/ biolreprod.108.074880

29. Mounib M. 1978. Cryogenic preservation of fish and mammalian spermatozoa. J Reprod Fertil 53: 13-18. doi: 10.1530/jrf.0.0530013

30. Neild D, Chaves G, Flores M, Mora N, Beconi M, Agüero A. 1999. Hypoosmotic test in equine spermatozoa. Theriogenology 51: 721-727. doi: 10.1016/S0093-691X(99)00021-7

31. Nouri H, Armin T, Zhandi M, Sadegui R. 2013. The effects of centrifuged egg yolk used with INRA plus soybean lecithin extender on semen quality to freeze miniature Caspian horse semen. J Equine Vet Sci 33: 1050-1053. doi: 10.1016/j.jevs.2013.03.184

32. Okamura N, Tajimat Y, Soejima A, Masudal H, Sugita Y. 1986. Sodium bicarbonate in seminal plasm stimulates the motility of mammalian spermatozoa through direct activation adenylate cyclase. J Biol Chem 260: 9699-9706.

33. Ortgies F, Klewitz J, Görgens A, Martinsson G, Sieme H. 2012. Effect of procaine, pentoxifylline and trolox on capacitation and hyperactivation of 
stallion spermatozoa. Andrologia 44: 130138. doi: 10.1111/j.1439-0272.2010.-01150.x

34. Padilla A, Foote R, 1991. Extender and centrifugation effects on the motility patterns of slow-cooled stallion spermatozoa. J Anim Sci 69: 3308-3313. doi: /1991.6983308x

35. Perrin D, Dempsey B. 1974. Buffers for $\mathrm{pH}$ and metal ion control. London: Chapman \& Hall. $176 \mathrm{p}$.

36. Pesch S, Bergmann M, Bostedt $H$. 2006. Determination of some enzymes and macro- and microelements in stallion seminal plasma and their correlations to semen quality. Theriogenology 66: 307313. doi: 10.1016/j.theriogenology.2005.11.015

37. Restrepo B, Cantero J, Montoya J. 2016. Efecto de la centrifugación sobre la integridad y la funcionalidad de espermatozoides equinos. Biotecnol Sector Agropec Agroind 14: 119-125. doi: 10.18684/BSAA(14)119-125

38. Restrepo G, Ocampo D, Velásquez A. 2013. Evaluación de la movilidad del semen criopreservado de caballos criollo colombiano por un sistema analizador de clase. Rev UDCA Act\&Div Cient 16: 445-450.

39. Restrepo G Usuga A, Montoya J, Celis A, Henao A. 2014. Evaluación de dos diluyentes para la criopreservación de semen de caballos de la raza criollo colombiano. Rev Lasallista Investig 11: 63-70.

40. Restrepo G, Zapata K, Rojano B. 2015. Evaluación de la capacidad antioxidante total del plasma seminal equino. Zootecnia Trop 31: 79-87.

41. Ribeiro B, Ramos R, Marques G, Gorzoni E, Trinca L, Dell'Aqua J, et al. 2015. Fixed-time insemination with frozen semen in mares: is it suitable for poorly fertile stallions? Theriogenology 83: 1389-1393. doi: 10.1016/j.theriogenology.2014.07.007
42. Sikka S. 2004. Role of oxidative stress and antioxidants in andrology and assisted reproductive technology. J Androl 25: 518. doi: 10.1002/j.1939-4640.2004.tb02751.x

43. Silva E, Cajueiro J, Silva S, Soares P, Guerra M. 2012. Effect of antioxidants resveratrol and quercetin on in vitro evaluation of frozen ram sperm. Theriogenology 277: 1722-1726. doi: 10.1016/j.theriogenology.2011.11.023

44. Tabassomi M, Alavi-Shoushtari S. 2013. Effects of in vitro copper sulphate supplementation on the ejaculated sperm characteristics in water buffaloes (Bubalus bubalis). Vet Res Forum 4: 31-36.

45. Waheed M, El-Bahr SM, Al-haider $A K$. 2013. Influence of seminal plasma antioxidants and osteopontin on fertility of the Arabian horse. J Equine Vet Sci 33: 705-709. doi: 10.1016/j.jevs.2012.11.006

46. Weilmeier D, Regenstein J. 2004. Antioxidant properties of phosphates and other additives during the storage of raw mackerel and lake trout. J Food Sci 69: 102-108. doi: 10.1111/j.1365-2621.2004.tb15498.x

47. Wnuk M, Lewinska A, Oklejewicz B, Bartosz G, Tischner M, BugnoPoniewierska M. 2010. Redox status of equine seminal plasma reflects the pattern and magnitude of DNA damage in sperm cells. Theriogenology 74: 16771684. doi: 10.1016/j.theriogenology.2010.07.007

48. Yildiz S, Öztürkler $Y$, Ari $U$, Lehimcioðlu N, Atakipi E, Kulaksiz R. 2015. The effects of L-ergothioneine, $\mathrm{N}$-acetylcystein and cystein on freezing of ram semen. Kafkas Univ Vet Fak Derg 21: 81-86. doi: 10.9775/kvfd.2014.11792 